\title{
Early experience and favorable clinical outcomes of everolimus-eluting bioresorbable scaffolds for coronary artery disease in Korea
}

Osung Kwon, Jung-Min Ahn, Do-Yoon Kang, Se Hun Kang, Pil Hyung Lee, Soo-Jin Kang, Seung-Whan Lee, Young-Hak Kim, Cheol Whan Lee, Seong-Wook Park, Duk-Woo Park, and Seung-Jung Park

Department of Cardiology, Asan Medical Center, University of Ulsan College of Medicine, Seoul, Korea

Received: November 14, 2016 Revised : February 14, 2017 Accepted: March 27, 2017

\section{Correspondence to} Duk-Woo Park, M.D. Department of Cardiology, Asan Medical Center, University of Ulsan College of Medicine, 88 Olympic-ro 43-gil, Songpa-gu, Seoul 05505, Korea Tel: +82-2-3010-3995 Fax: +82-2-475-6898 E-mail:dwpark@amc.seoul.kr
Background/Aims: Compared with metallic drug-eluting stents (DES), bioresorbable vascular scaffolds (BVS) may further improve long-term outcomes of percutaneous coronary intervention (PCI) in patients with coronary artery disease. We report our early experience with BVS in Korea.

Methods: We evaluated 105 consecutive patients with BVS implanted at Asan Medical Center, Korea between October 21, 2015 and June 3, 2016. Angiographic results, and in-hospital and 6-month clinical outcomes were assessed.

Results: A total of 134 BVS were implanted to treat 115 lesions. The mean age was $62 \pm 10.5$ years; 85 patients $(81 \%)$ were males, 26 patients $(25 \%)$ were presented with acute coronary syndrome. Among 115 lesions treated with BVS, 76 (66.1\%) were B2/C type, 27 (23.5\%) were bifurcation lesions, and four (3.5\%) were chronic total occlusion. Pre-dilation and post-dilation using high-pressure non-compliant balloon was performed in 104 lesions (90.4\%) and 113 lesions (98.2\%), respectively. During the procedure, intravascular imaging was used for all patients $(100 \%$; intravascular ultrasound 89 and optical coherence tomography 40 patients). Device success rate was 100\%. In-segment and in-scaffold acute again were $1.1 \pm 0.6$ and 1.3 $\pm 0.5 \mathrm{~mm}$, respectively. Periprocedural myocardial infraction occurred in four patients (3.8\%). No deaths, stent thrombosis, or urgent revascularizations occurred either during hospitalization or the follow-up period.

Conclusions: In this single-center experience, implantation of BVS with intravascular imaging support was feasible and early clinical outcomes were excellent. Evaluation of long-term efficacy and safety of BVS and its feasibility in clinical use for a broader range of lesions is warranted.

Keywords: Absorbable implants; Bioresorbable vascular scaffolds; Percutaneous coronary intervention

\section{INTRODUCTION}

For the past decade, drug-eluting stents (DES) has been established as the standard device for percutaneous coronary intervention (PCI) for ischemic heart disease [1].
With continuous advancements in drug, polymer, and stent design, contemporary DES have been associated with better clinical outcomes than have either bare-metal stents or first-generation DES and are currently being widely used in unrestricted clinical and anatomical set- 
tings [2].

However, ongoing risks of late stent thrombosis (ST) and restenosis limit their long-term safety and efficacy. In addition, permanent metallic stents itself might be related to persistent inflammation, stent fracture, neoatherosclerosis and loss of normal vessel curvature, and also could disrupt late luminal enlargement, adaptive shear stress and natural restoration of vasomotion [3,4].

These inherent limitations of metallic DES have prompted a development of novel concept of devices such as bioresorbable vascular scaffolds (BVS) that provide mechanical support and drug-delivery for approximately 1 year and then are completely bioresorbable over several years [5]. Several versions of BVS have been developed and tested in preclinical or clinical setting on humans. The Absorb BVS (Abbott Vascular, Santa Clara, CA, USA) is the first commercially available drug-eluting bioresorbable scaffold consisting of a $150-\mu \mathrm{m}$-thick bioresorbable poly (L-lactide) scaffold with a $7-\mu \mathrm{m}$ thick bioresorbable poly (D,L-lactide) coating, which elutes everolimus. Several randomized clinical trials showed non-inferior clinical outcomes of BVS as compared with contemporary metallic DES [6-11]. However, there are still lacking with regard to information on clinical experience and outcomes after BVS use in "real-world" patients with diverse clinical and angiographic characteristics. Herein, we report the first experience of BVS implantation in Korea, which was recently approved for use since October 21, 2015.

\section{METHODS}

\section{Study population and procedures}

The study population included consecutive patients who underwent PCI with at least one BVS for stable angina or acute coronary syndrome (ACS) at Asan Medical Center (Seoul, Korea) between October 21, 2015, and June 3, 2016. Selection of Absorb BVS for the PCI procedure was at the discretion of the attending physicians. This study was approved by the Institutional Review Board of Asan Medical Center (2016-0006), and all patients provided written informed consent. There was no industry involvement in the design, conduct, or analysis of the study.

The interventional cardiologists complied with the in- structions for optimal implantation of the Absorb BVS. The procedure was performed according to the PSP implant technique ( $\mathrm{P}$, prepare the lesion; $\mathrm{S}$, size appropriately; P, post-dilate). For appropriate lesion preparation, a judicious use of pre-dilatation, cutting/scoring balloons and rotational atherectomy was applied, as appropriately [12]. Pre-dilation was routinely performed with a non-compliant balloon with 1:1 balloon-to-artery ratio. During the procedure, intravascular imaging with grayscale-intravenous ultrasound (IVUS) or optical coherence tomography (OCT) was routinely used for accurate sizing of the BVS. A standard dose of intracoronary nitroglycerin was administered prior to determining the reference vessel diameter (RVD). The size of the scaffold was adequately matched to the size of the vessel using the information obtained during vessel preparation and by intravascular imaging. Scaffolds were not recommended for vessel size $>4.0 \mathrm{~mm}$ diameter by quantitative coronary analysis (QCA), IVUS or OCT. The scaffold was deployed slowly by pressurizing the delivery system in 2 atm increments over 5 seconds, until it was completely expanded. The target deployment pressure was maintained for 30 seconds, or as long as tolerated by the patient. After BVS implantation, high-pressure (> $16 \mathrm{~atm}$ ) post-dilatation with a noncompliant balloon up to $0.5 \mathrm{~mm}$ larger than the nominal scaffold diameter was routinely performed to achieve full expansion. The expansion was limited to a diameter no more than 0.5 $\mathrm{mm}$ larger than the nominal scaffold diameter to avoid strut fracture. After high-pressure dilation, scaffold struts were visualized and strut apposition was assessed by IVUS or OCT. After the procedure, dual antiplatelet therapy (DAPT) with aspirin and an adenosine diphosphate receptor antagonist (clopidogrel, ticagrelor, or prasugrel) was prescribed for at least 12 months. Cilostazol was added to DAPT regimens at the physician's discretion.

\section{Quantitative coronary angiography}

Coronary angiograms were digitally recorded at baseline and immediately after the procedure, and were assessed offline in the angiographic core laboratory (Asan Medical Center, Seoul, Korea) using an automated edge-detection system (CAAS-5, Pie-Medical, Best, the Netherlands) by experienced assessors unaware of the allocated stent. All measurements were performed 
on cineangiograms recorded after the intracoronary administration of nitroglycerin. Standard qualitative and quantitative analyses and definitions were used for angiographic analysis. The reference diameter was determined by interpolation. Results of procedural QCA were reported in-device (within the scaffold, measured edge-to-edge) and in-segment (within the scaffold plus the $5-\mathrm{mm}$ proximal and distal margins). Angiographic variables included absolute lesion length, scaffold length, RVD, minimum lumen diameter (MLD), percent diameter stenosis (DS), and immediate acute gain. The acute gain was defined as the difference between pre-procedure MLD and post-procedure MLD within the scaffold or segment.

\section{Clinical outcomes and definition}

Outcomes measured for composite clinical endpoints were death (cardiac vs. non-cardiac), myocardial infarction (MI; Q-wave vs. non-Q-wave), target-lesion revascularization (TLR), target-vessel revascularization (TVR), non-TVR, and all coronary revascularizations. Composite clinical endpoints were target-vessel failure (TVF; a composite of cardiac death, any MI, or ischemia-driven TVR), target-lesion failure (TLF; a composite of cardiac death, target-vessel MI, and ischemia-driven TLR), major adverse cardiac events (a composite of cardiac death, MI, or TLR), device-oriented combined endpoint (a composite of cardiac death, target-vessel MI, and TLR), and patient-oriented combined endpoint (a composite of all-cause death, any MI, and any repeat revascularization.).

All deaths were considered to have been from cardiac causes unless a noncardiac cause could be identified. Diagnosis of acute MI was made by Society for Cardiovascular Angiography and Interventions guideline [13]. Revascularization of the target lesion and vessel was considered ischemia driven if there was stenosis of at least $50 \%$ of the diameter of the treated lesion or vessel by QCA at the independent core laboratory in the presence of ischemic signs (i.e., positive functional tests) or symptoms, or a target vessel (or lesion) DS of $70 \%$ or greater with or without documented ischemia. Scaffold thrombosis was assessed using Academic Research Consortium criteria [14]. Device success was defined as a successful delivery and deployment of scaffolds at the intended target lesion and successful withdrawal of the delivery system with attainment of final in-scaffold residual stenosis of less than $30 \%$ by QCA or by visual estimation if QCA was unavailable [15].

\section{Statistical analysis}

Baseline characteristics, including patient demographics, risk factors, clinical presentation, cardiac status, in-hospital medications, and anatomic/procedural characteristics were described. Categorical variables were expressed as number and percentages; continuous variables were expressed as mean \pm standard deviation (SD). The numbers and percentages of patient in-hospital and 6-month follow-up outcomes were recorded, and cumulative survival was estimated by Kaplan-Meier event rate plots. All statistical analyses were performed with of IBM SPSS version 21 (IBM Corp., Armonk, NY, USA).

\section{RESULTS}

\section{Baseline characteristics and procedural results}

From October 21, 2015, to June 3, 2016, a total of 105 patients who received PCI with BVS were included in the current analysis. Baseline demographics and clinical characteristics of patients are shown in Table 1 . The mean age was 62 years and $81 \%$ were male. One-thirds of patients had diabetes and $8 \%$ had previous history of PCI. Three-quarters of patients were presented with stable angina or silent ischemia and the remaining was presented with ACS; nine patients underwent BVS implantation during the primary PCI for STEMI. The mean ejection fraction was $61 \%$ and $44 \%$ had multi-vessel disease. After BVS implantation, aspirin and $\mathrm{P}_{2} \mathrm{Y}_{12}$ inhibitors were prescribed in $98.1 \%$ and $100 \%$ (clopidogrel 86\% and ticagrelor 14\%), respectively. More than $90 \%$ of patients received cilostazol add-on DAPT.

In 105 patients, 138 lesions were treated with BVS or stent implantation during the PCI, in which 115 lesions were treated with BVS and the remaining 23 lesions were treated with DES. A total of 25 patients received two or more BVS; 21 patients with two BVS, three patents with three BVS, and one patient with four BVS. In 19 patients, BVS and DES were implanted simultaneously in the different lesions. There was no hybrid approach in this series. Baseline lesion and procedural characteristics with regard to BVS implantation are shown in 
Table 2. More than half of lesions were located in the left anterior descending artery and had complex lesion types of $\mathrm{B} 2$ or $\mathrm{C}$ according to the AHA/ACC classifica- tion. Most of lesions were de novo lesions and 23.5\% were true bifurcation lesions. In all bifurcation lesions, the provisional strategy was employed. Simple crossover

Table 1. Baseline demographics and clinical characteristics

\begin{tabular}{|c|c|}
\hline Variable & Overall patient $(n=105)$ \\
\hline \multicolumn{2}{|l|}{ Demographics } \\
\hline Age, yr & $61.5 \pm 10.5$ \\
\hline Male sex & $85(81)$ \\
\hline Body-mass index, $\mathrm{kg} / \mathrm{m}^{2}$ & $24.6 \pm 2.6$ \\
\hline \multicolumn{2}{|l|}{ Risk factor and clinical history } \\
\hline Hypertension & $66(62.9)$ \\
\hline Hyperlipidemia & $83(79.0)$ \\
\hline \multicolumn{2}{|l|}{ Diabetes } \\
\hline Any & $29(27.6)$ \\
\hline Insulin-requiring & $4(3.8)$ \\
\hline Current smoker & $27(25 \cdot 7)$ \\
\hline Family history of coronary artery disease & $19(18.1)$ \\
\hline Prior percutaneous coronary intervention & $8(7.6)$ \\
\hline Prior coronary artery bypass grafting & $3(2.9)$ \\
\hline Prior cerebrovascular accident & $2(1.9)$ \\
\hline Peripheral arterial disease & $1(1.0)$ \\
\hline Chronic lung disease & $3(2.9)$ \\
\hline Chronic renal failure & $1(1.0)$ \\
\hline \multicolumn{2}{|c|}{ Clinical indication for percutaneous coronary intervention } \\
\hline Stable angina or silent ischemia & $79(75 \cdot 3)$ \\
\hline Unstable angina & $9(8.6)$ \\
\hline Non-ST-segment elevation MI & $8(7.6)$ \\
\hline ST-segment elevation MI & $9(8.6)$ \\
\hline Left ventricular ejection fraction, $\%^{\mathrm{a}}$ & $61.3 \pm 6.5$ \\
\hline Multivessel disease & $46(43.8)$ \\
\hline \multicolumn{2}{|l|}{ Concomitant medication at in-hospital or discharge } \\
\hline Aspirin & $103(98.1)$ \\
\hline \multicolumn{2}{|l|}{ Type of $\mathrm{P}_{2} \mathrm{Y}_{12}$ inhibitor } \\
\hline Clopidogrel & $90(85 \cdot 7)$ \\
\hline Ticagrelor & $15(14 \cdot 3)$ \\
\hline Prasugrel & o \\
\hline Cilostazol & $97(92.4)$ \\
\hline$\beta$-Blocker & $77(73 \cdot 3)$ \\
\hline Calcium channel blocker & $67(63.8)$ \\
\hline Statin & $102(97.1)$ \\
\hline ACE inhibitor or angiotensin-II receptor blocker & $30(28.6)$ \\
\hline
\end{tabular}

Values are presented as mean \pm standard deviation or number (\%).

MI, myocardial infarction; ACE, angiotensin converting enzyme.

${ }^{\text {a }}$ Measurement of left ventricular ejection fraction was available in 50 patients. 
stenting was performed and in only one case, after BVS implantation in the main branch, balloon dilatation for the side branch was done. For all five diffuse long lesions ( $\geq 40 \mathrm{~mm}$ ), scaffold implantation was performed, allowing minimal overlapping using intravascular im- age devices. Average number of scaffolds implanted and total scaffolds length per lesion were 1.16 and $23.3 \mathrm{~mm}$, respectively. For BVS implantation, the majority of lesions were predilated (90.4\%) and also post high-pressure balloon dilation was performed in 113 lesions

Table 2. Lesion and procedural characteristics

\begin{tabular}{|c|c|}
\hline Variable & BVS-treated lesion $(n=115)$ \\
\hline \multicolumn{2}{|l|}{ Lesion characteristic } \\
\hline \multicolumn{2}{|l|}{ Treated vessel } \\
\hline Left main coronary artery & $2(1.7)$ \\
\hline Left anterior descending & $72(62.6)$ \\
\hline Left circumflex & $21(18.3)$ \\
\hline Right coronary artery & $20(17 \cdot 4)$ \\
\hline \multicolumn{2}{|l|}{ ACC/AHA lesion type } \\
\hline A & $5(4 \cdot 3)$ \\
\hline $\mathrm{B} 1$ & $34(29.6)$ \\
\hline $\mathrm{B} 2$ & $12(10.4)$ \\
\hline $\mathrm{C}$ & $64(55 \cdot 7)$ \\
\hline De novo lesion & $114(99.1)$ \\
\hline In-stent restenosis & $1(0.9)$ \\
\hline Calcification, severe & $3(2.6)$ \\
\hline Long lesion, $\geq 40 \mathrm{~mm}$ & $5(4 \cdot 3)$ \\
\hline Chronic total occlusion & $4(3.5)$ \\
\hline Bifurcated lesion & $27(23.5)$ \\
\hline Thrombus present & $2(1.4)$ \\
\hline \multicolumn{2}{|l|}{ Procedure characteristic } \\
\hline Total number of scaffolds, /patient & $134(1.28)$ \\
\hline Average number of scaffolds implanted, /lesion & $1.16 \pm 0.37$ \\
\hline Total scaffolds length, per lesion, mm & $23.3 \pm 4.8$ \\
\hline Pre-dilation & $104(90.4)$ \\
\hline Post-dilation using high-pressure balloon & $113(98.2)$ \\
\hline Maximum scaffold diameter, $\mathrm{mm}^{\mathrm{a}}$ & $3.67 \pm 0.35$ \\
\hline Ratio of maximum scaffold diameter to vessel diameter & $1.22 \pm 0.14$ \\
\hline Maximum balloon pressure, atm ${ }^{\mathrm{a}}$ & $21.41 \pm 4.48$ \\
\hline Intravascular imaging guidance (IVUS or OCT) & $115(100)$ \\
\hline \multicolumn{2}{|l|}{ IVUS use } \\
\hline Pre & $89(77.4)$ \\
\hline Post & $84(73.0)$ \\
\hline \multicolumn{2}{|l|}{ OCT use } \\
\hline Pre & $37(32.2)$ \\
\hline Post & $40(34.8)$ \\
\hline Procedural duration, min & $58.4 \pm 24.4$ \\
\hline Device success/patient & $105(100)$ \\
\hline
\end{tabular}

Values are presented as number (\%) or mean \pm standard deviation.

BVS, bioresorbable vascular scaffold; ACC/AHA, American College of Cardiology/American Heart Association; IVUS, intravascular ultrasound; OCT, optical coherence tomography,

${ }^{a}$ Maximum diameter or pressure of the predilation balloon, the scaffold delivery-system balloon, or the post-dilation balloon. 


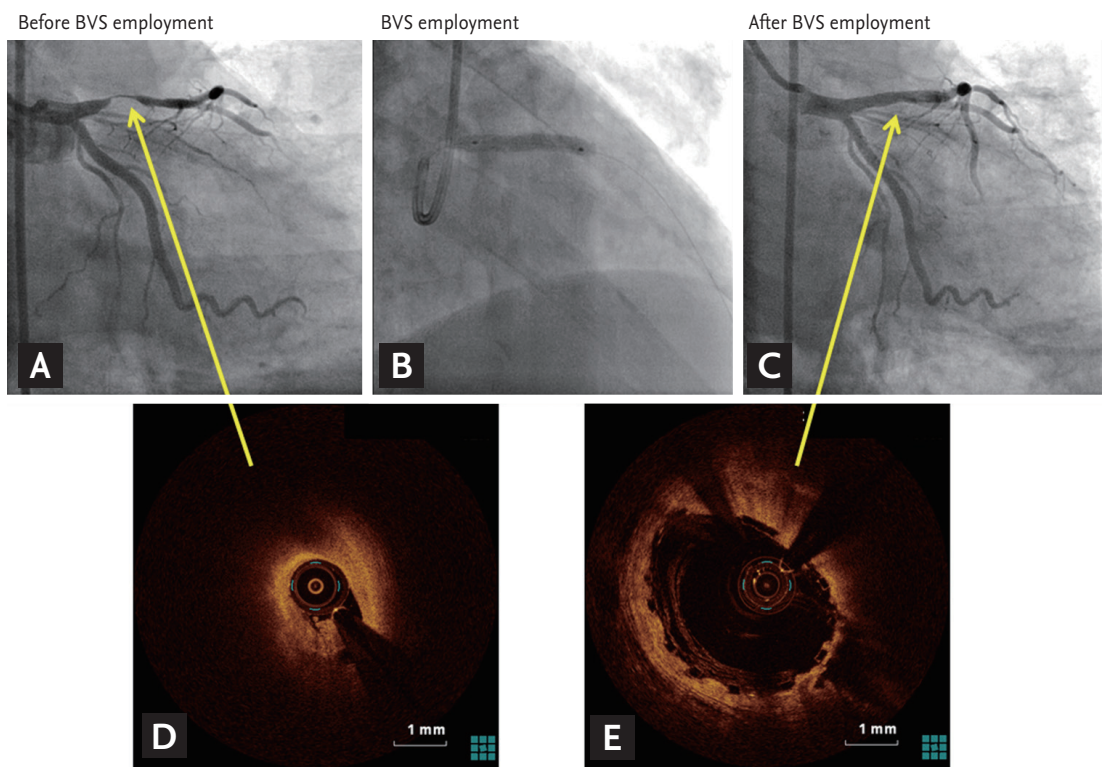

Figure 1. Angiographic and optical coherence tomographic findings of a representative patient with successful bioresorbable vascular scaffold (BVS) implantation. Successful BVS employment in a patient with ST-segment elevation myocardial infarction. (A) Angiography showed near total occlusion of left anterior descending artery. (B) BVS employment was performed. (C) Angiography revealed successful revascularization after BVS employment. (D) Optical coherence tomography (OCT) demonstrated thrombus formation on ruptured plaque. (E) Deeply embedded BVS in the arterial wall following high-pressure post-dilation was seen on OCT. Yellow arrows indicate lesions where OCT was performed.

Table 3. Quantitative coronary angiography results of scaffold implantation

\begin{tabular}{|c|c|}
\hline Variable & $\begin{array}{c}\text { BVS-treated lesion } \\
(\mathrm{n}=115)\end{array}$ \\
\hline \multicolumn{2}{|l|}{ Before the procedure } \\
\hline Reference vessel diameter, $\mathrm{mm}$ & $3.1 \pm 0.4$ \\
\hline Minimum luminal diameter, $\mathrm{mm}$ & $1.2 \pm 0.5$ \\
\hline Diameter stenosis, \% & $60.5 \pm 17.1$ \\
\hline Lesion length, mm & $20.0 \pm 9.0$ \\
\hline \multicolumn{2}{|l|}{ After the procedure } \\
\hline \multicolumn{2}{|l|}{ In-device measures } \\
\hline Minimum luminal diameter, mm & $2.5 \pm 0.3$ \\
\hline Diameter stenosis, \% & $15.5 \pm 7 \cdot 7$ \\
\hline Acute gain ${ }^{\mathrm{a}}, \mathrm{mm}$ & $1.3 \pm 0.5$ \\
\hline \multicolumn{2}{|l|}{ In-segment measures } \\
\hline Minimum luminal diameter, mm & $2.3 \pm 0.4$ \\
\hline Diameter stenosis, \% & $20.1 \pm 8.3$ \\
\hline Acute gain ${ }^{\mathrm{a}}, \mathrm{mm}$ & $1.1 \pm 0.6$ \\
\hline
\end{tabular}

Values are presented as mean \pm standard deviation.

${ }^{a}$ Acute gain was defined as the change in the minimal lumen diameter from baseline to the final procedural angiogram.

(98.2\%). During the procedure, intravascular imaging guidance were used in all lesions (IVUS $77.4 \%$ and OCT $34.8 \%)$. Overall, the BVS implantation was successful in all patients (device success rate of 100\%). Representative case who underwent successful BVS implantation was illustrated in Fig. 1.

\section{Angiographic outcomes}

Quantitative angiographic results at baseline and immediately post-procedure are shown in Table 3. The mean lesion length was $20.0 \mathrm{~mm}$ and DS was $61 \%$. After the procedure, in-device and in-segment minimum luminal diameter $(\mathrm{MLD})$ was $2.5 \pm 0.3$ and $2.3 \pm 0.4 \mathrm{~mm}$, respectively. In-device and in-segment acute gain was 1.3 \pm 0.5 and $1.1 \pm 0.6 \mathrm{~mm}$, respectively. Pre- and post-proe cedural in-segment MLD changes and in-segment acute gain after procedure are shown in Fig. 2.

\section{Clinical outcomes}

Mean follow-up was 105.4 \pm 74.9 days, 1-month follow-up was available in all patients. Forty-three patients had at least 6-month follow-up period and clinical follow-up at 6-month was available for all period-eligible patients. The in-hospital and cumulative clinical events at 6-month are summarized in Table 4. No cases of mortality, scaffold thrombosis, or urgent revascularization occurred during the hospital stay or during the 6-month follow-up. Periprocedural non-Q-wave MI occurred in four patients (3.8\%) (Fig. 3). Overall, a device-oriented or patient-oriented clinical endpoint occurred in four patients (3.8\%), due to periprocedural MI (Fig. 3). Clinical and procedural characteristics of the four patients who 




Figure 2. Minimal lumen diameter (MLD, in-segment) at pre- and post-scaffold implantation and acute gain of lumen diameter.

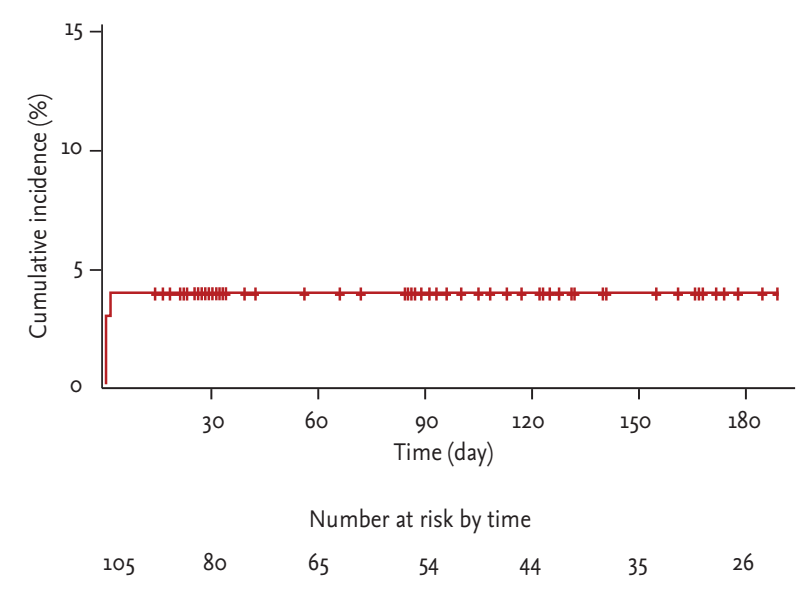

Figure 3. Kaplan-Meier cumulative incidence of major cardiac adverse events.

Table 4. Clinical events during the index hospitalization and follow-up

\begin{tabular}{|c|c|c|}
\hline Variable & In-hospital ( $\mathrm{n}=105)$ & 6 Months $(n=43)$ \\
\hline \multicolumn{3}{|l|}{ Outcome } \\
\hline Follow-up rate (no./period-eligible patients) & $105(100)$ & $43(100)$ \\
\hline All death & o & o \\
\hline Non-cardiac death & 0 & o \\
\hline Cardiac death & o & 0 \\
\hline Any myocardial infarction & $4(3.8)$ & $4(3.8)$ \\
\hline Q-wave & o & o \\
\hline Non-Q-wave & $4(3.8)$ & $4(3.8)$ \\
\hline All revascularization & o & o \\
\hline Target vessel revascularization & 0 & 0 \\
\hline Target lesion revascularization & o & o \\
\hline Target vessel failure ${ }^{\mathrm{a}}$ & $4(3.8)$ & $4(3.8)$ \\
\hline Target lesion failure ${ }^{\mathrm{b}}$ & $4(3.8)$ & $4(3.8)$ \\
\hline Major cardiac adverse event ${ }^{c}$ & $4(3.8)$ & $4(3.8)$ \\
\hline Device-oriented combined endpoint ${ }^{\mathrm{d}}$ & $4(3.8)$ & $4(3.8)$ \\
\hline Patient-oriented combined endpoint $\mathrm{e}^{\mathrm{e}}$ & $4(3.8)$ & $4(3.8)$ \\
\hline \multicolumn{3}{|l|}{ ARC scaffold thrombosis } \\
\hline \multicolumn{3}{|l|}{ Definite } \\
\hline Acute (o-1 day) & 0 & O \\
\hline Subacute (2-30 day) & 0 & o \\
\hline Late (31-18o day) & o & o \\
\hline Definite or probable & 0 & 0 \\
\hline
\end{tabular}

Values are presented as number (\%).

ARC, Academic Research Consortium.

${ }^{a}$ Cardiac death, any myocardial infarction, or ischemia-driven target vessel revascularization.

${ }^{\mathrm{b}}$ Cardiac death, target-vessel myocardial infarction, or ischemia-driven target lesion revascularization.

${ }^{\mathrm{c}}$ Cardiac death, myocardial infarction, or ischemic-driven target lesion revascularization.

${ }^{\mathrm{d}}$ Cardiac death, target-vessel myocardial infarction, and target-lesion revascularization.

${ }^{\mathrm{e}}$ All-cause death, any recurrent myocardial infarction, and any repeat revascularization. 
Table 5. Clinical, angiographic, and procedural characteristics of patients who experienced periprocedural myocardial infarction

\begin{tabular}{|c|c|c|c|c|}
\hline Case & Patient 1 & Patient 2 & Patient 3 & Patient 4 \\
\hline Age, yr & 67 & 54 & 49 & 69 \\
\hline Sex & Male & Male & Male & Male \\
\hline Hypertension & Yes & No & Yes & Yes \\
\hline Diabetes mellitus & No & No & No & No \\
\hline Hyperlipidemia & Yes & Yes & Yes & Yes \\
\hline Current smoking & No & Yes & Yes & Yes \\
\hline Clinical presentation & UA & NSTEMI & STEMI & STEMI \\
\hline $\begin{array}{l}\text { Peak creatine kinase-MB/troponin I, } \\
\mathrm{ng} / \mathrm{mL}\end{array}$ & $244.2 / 61.3$ & $436.3 / 157.8$ & $287.1 / 106.2$ & $353 / 216.0$ \\
\hline Disease extent & 2 Vessel & 1 Vessel & 1 Vessel & 1 Vessel \\
\hline No. of treated lesion & 2 & 1 & 1 & 1 \\
\hline Total no. of BVS & 3 & 1 & 1 & 1 \\
\hline \multicolumn{5}{|l|}{ Treated lesion \#1 } \\
\hline Location & 1st OM & Proximal LAD & Mid RCA & Proximal LCX \\
\hline Bifurcation & No & Yes & No & No \\
\hline ACC/AHA & $\mathrm{B} 1$ & $\mathrm{C}$ & $\mathrm{B} 2$ & $\mathrm{~B} 2$ \\
\hline Thrombus & No & Yes & No & No \\
\hline Treatment device & $\begin{array}{l}3.0 \times 18 \\
\text { Absorb }\end{array}$ & $\begin{array}{l}3.5 \times 23 \\
\text { Absorb }\end{array}$ & $\begin{array}{l}3 \cdot 5 \times 28 \\
\text { Absorb }\end{array}$ & $\begin{array}{l}3 \cdot 5 \times 23 \\
\text { Absorb }\end{array}$ \\
\hline \multicolumn{5}{|l|}{ Treated lesion \#2 } \\
\hline Location & Proximal LAD & - & - & - \\
\hline Bifurcation & Yes & - & - & - \\
\hline ACC/AHA & $\mathrm{C}$ & - & - & - \\
\hline Thrombus & Yes & - & - & - \\
\hline Treatment device & $\begin{array}{c}4.0 \times 38 \\
\text { Xience Alpine }\end{array}$ & - & - & - \\
\hline Comment & $\begin{array}{c}\text { Lesion 1: side branch } \\
\text { (1st diagonal) } \\
3.5 \times 18 \text { Xience Alpine } \\
\text { implanted }\end{array}$ & - & $\begin{array}{l}\text { Lesion 1: total } \\
\text { occlusion }\end{array}$ & - \\
\hline
\end{tabular}

UA, unstable angina; NSTEMI, non-ST-segment elevation myocardial infarction; STEMI, ST-segment elevation myocardial infarction; MB, myocardial band; BVS, bioresorbable vascular scaffold; OM, obtuse marginal; LAD, left anterior descending; RCA, right coronary artery; LCX, left circumflex; ACC/AHA, American College of Cardiology/American Heart Association.

experienced periprocedural MI are described in Table 5.

\section{DISCUSSION}

This study is the first report on the feasibility of BVS implantation and the safety/efficacy of BVS for patients with significant coronary artery disease (CAD) in Korea. Our initial experience showed that BVS implantation was technically feasible in all patients and early clinical outcomes were also excellent without scaffold thrombosis, urgent revascularization, or mortality.

Conventional metallic stents are associated with an ongoing long-term concerns of TLF due to very late restenosis and thrombosis. Such failure may be caused by the persistence of rigid metallic frame that straightens the normal vascular curvature and cages the external dimension of the vessel and interferes with vasoregulation and chronic adaptive vascular responses [3-5]. In addition, permanent metallic stent itself can be a nidus for 
chronic inflammation or neoatherosclerosis and poses a risk of device fracture. Thus, BVS were developed to overcome such inherent limitations; BVS restore cyclic pulsatility by 6 months and vasomotor responses by 12 months, and scaffold resorption results in increased luminal dimensions due to adaptive remodeling of the external elastic membrane and plaque regression $[16,17]$, which could thereby improve long-term outcomes compared with contemporary metallic DES.

Previous several real-world registry data showed an excellent procedural success rate $[18,19]$. Likewise, in our first experience of BVS implantation, successful scaffold implantation was achieved for all patients. In this series, the angiographic acute gain in scaffold was $1.3 \pm 0.5 \mathrm{~mm}$, compatible to a previous "real-world" setting report of $1.37 \pm 0.53 \mathrm{~mm}$ [18]. Acute gain after BVS implantation tends to be lower than that of conventional DES implantation $[7,8]$. This could be explained by differences in both the mechanical properties of the devices, and initial implantation strategies, which is emphasized with adequate pre-dilation and optimal expansion with careful high-pressure post dilation.

Considering thick strut thickness of BVS, a potential concern exists that it may be associated with a higher risk of periprocedural myocardial injury or MI compared to contemporary metallic DES. Thicker strut of BVS may be associated with a higher propensity of distal embolization and side branch occlusion which are prominent mechanisms of periprocedural MI [20] and also aggressive pre- and post-dilation for meticulous lesion preparation and scaffold full expansion with good apposition could lead to distal embolization of plaque debris or thrombus. Reports that evaluated the potential risk of periprocedural MI associated with BVS implantation have yielded conflicting results $[7,9,20,21]$. Further study is needed to determine whether the thicker strut dimensions of BVS are directly related to an increase in the rate of periprocedural myonecrosis and to assess prognostic impact of periprocedural MI on BVS outcomes.

Previous randomized clinical trials and real-world setting investigations showed non-negligible, higher rates of early scaffold thrombosis than contemporary metallic DES. Concerns have been raised that the BVS is more thrombogenic than DES. In the landmark ABSORB III trial [7], subacute thrombosis between 1 and 30 days after implantation was more common with the Absorb BVS than with everolimus-eluting stents (0.9\% vs. $0.1 \%, p=$ 0.04). Several clinical registries also indicated a scaffold thrombosis rate of approximately $1 \%$ to $2 \%$ at 30 days and $2 \%$ to $3 \%$ at 6 months $[18,19,22]$. Theoretically, it is postulated that thicker BVS struts $(150 \mu \mathrm{m})$ could potentially cause microscopic intra-coronary flow disturbances, which enhance thrombus formation and potentially early ST prior to neointimal coverage [3]. Practically, the bulkier BVS device is more difficult to employ and, as a result, is more vulnerable to procedural issue such as dissection, incomplete stent apposition, or incomplete stent expansion [23]. Thick strut and its-related possibility of early scaffold thrombosis strongly advocates the adequate lesion preparation before scaffold placement, optimal sizing, and/or post-dilatation with the use of a non-compliant balloon, such as "PSP implant technique" [24]. Although clinical follow-up was still limited, in our registry, no patients developed ST at 30 days and 6 months; some patients have not yet completed the 6-month follow-up. In our registry, the majority of the patients received pre- and post-balloon dilatation using non-compliant balloon and also procedural optimization using intravascular imaging tools was routinely performed. On contrary to our report, the previous studies demonstrated lower rates of intracoronary image use less than $20 \%[18,19]$ even as low as $1 \%[22]$. According to a recent study evaluating clinical utility of OCT, despite achieving angiographic success in all BVS implantation, further optimization was required in over a quarter of patients on the basis of OCT findings [25]. Intracoronary image could identify procedural factors for ST such dissection, incomplete stent apposition, incomplete stent expansion, resulting in optimal stent employment. Our results might imply the necessity of routine intracoronary image during PCI with the BVS to prevent ST, the most feared event. Further studies are warranted to evaluate the clinical impact of the invasive image modalities for BVS implantation.

Of note, we prescribed triple antiplatelet agents in more than $90 \%$ patients, which noticeably differed from the previous studies equipped with dual antiplatelet strategy. Previous data regarding triple therapy for DES implantation demonstrated the effect reducing ST [26]. The aggressive antiplatelet strategy in the registry might contribute to no ST events during the follow-up peri- 
od. The optimal regimen and duration of antiplatelet agents following PCI with BVS is unknown and thus should be investigated in the future studies.

Several limitations of our study should be noted. First, this is a single-arm registry study with no comparator group. Thus, it was not able to compare the device with contemporary devices. Second, the participants were a relatively small series enrolled at a single center, which limits the generalizability of the findings. Third, the choice of a device for BVS implantation to treat obstructive $\mathrm{CAD}$ was subject to selection bias. Finally, follow-up was limited in duration and by the small patient sample. Therefore, long-term follow-up of our study patients and conduct of large-scale clinical trials or patient series are required to confirm the long-term safety and efficacy of BVS.

In conclusion, in this first-experience report of BVS in Korea, which included patients with relatively unselective clinical characteristics and high-risk lesions, the use of Absorb BVS demonstrated excellent procedural feasibility and acceptable clinical outcomes at early-term follow-up. A strategy of scrupulous lesion preparation and intracoronary image-guided post-balloon dilation in combination with aggressive antiplatelet therapy might be associated with these encouraging early results. Further extended investigations are warranted to establish long term safety and efficacy in all comer population.

\section{KEY MESSAGE}

1. Although data from randomized trials raise concerns about stent thrombosis (ST) after bioresorbable vascular scaffold (BVS) implantation, in this first-experience report of BVS in Korea, using a real-world all-comer registry, the use of Absorb BVS demonstrated excellent procedural feasibility and favorable clinical outcomes without ST at early-term follow-up.

2. Strategy of meticulous lesion preparation and intracoronary image-guided post-balloon dilation, complying with PSP implant technique (Preparing the lesion adequately before scaffold placement; Sizing appropriately; Post-dilatation with the use of a non-compliant balloon) may be associated with these encouraging early results.

3. Aggressive antiplatelet therapy including ci- lostazol add-on dual antiplatelet therapy might contribute to preventing ST following BVS implantation, which should be investigated in further studies.

\section{Conflict of interest}

No potential conflict of interest relevant to this article was reported.

\section{Acknowledgments}

This work was supported by the Cardio Vascular Research Foundation, Seoul, Korea.

\section{REFERENCES}

1. Authors/Task Force members, Windecker S, Kolh P, et al. 2014 ESC/EACTS guidelines on myocardial revascularization: the task force on myocardial revascularization of the European Society of Cardiology (ESC) and the European Association for Cardio-Thoracic Surgery (EACTS) Developed with the special contribution of the European Association of Percutaneous Cardiovascular Interventions (EAPCI). Eur Heart J 2014;35:2541-2619.

2. Marroquin OC, Selzer F, Mulukutla SR, et al. A comparison of bare-metal and drug-eluting stents for off-label indications. N Engl J Med 2008;358:342-352.

3. Onuma Y, Serruys PW. Bioresorbable scaffold: the advent of a new era in percutaneous coronary and peripheral revascularization? Circulation 2011;123:779-797.

4. Otsuka F, Vorpahl M, Nakano M, et al. Pathology of second-generation everolimus-eluting stents versus first-generation sirolimus- and paclitaxel-eluting stents in humans. Circulation 2014;129:211-223.

5. Serruys PW, Garcia-Garcia HM, Onuma Y. From metallic cages to transient bioresorbable scaffolds: change in paradigm of coronary revascularization in the upcoming decade? Eur Heart J 2012;33:16-25.

6. Serruys PW, Chevalier B, Dudek D, et al. A bioresorbable everolimus-eluting scaffold versus a metallic everolimus-eluting stent for ischaemic heart disease caused by de-novo native coronary artery lesions (ABSORB II): an interim 1-year analysis of clinical and procedural secondary outcomes from a randomized controlled trial. Lancet 2015;385:43-54. 
7. Ellis SG, Kereiakes DJ, Metzger DC, et al. Everolimus-eluting bioresorbable scaffolds for coronary artery disease. N Engl J Med 2015;373:1905-1915.

8. Kimura T, Kozuma K, Tanabe K, et al. A randomized trial evaluating everolimus-eluting Absorb bioresorbable scaffolds vs. everolimus-eluting metallic stents in patients with coronary artery disease: ABSORB Japan. Eur Heart J 2015;36:3332-3342.

9. Gao R, Yang Y, Han Y, et al. Bioresorbable vascular scaffolds versus metallic stents in patients with coronary artery disease: ABSORB China Trial. J Am Coll Cardiol 2015;66:2298-2309.

10. Stone GW, Gao R, Kimura T, et al. 1-Year outcomes with the Absorb bioresorbable scaffold in patients with coronary artery disease: a patient-level, pooled meta-analysis. Lancet 2016;387:1277-1289.

11. Cassese S, Byrne RA, Ndrepepa G, et al. Everolimus-eluting bioresorbable vascular scaffolds versus everolimus-eluting metallic stents: a meta-analysis of randomised controlled trials. Lancet 2016;387:537-544.

12. Iqbal J, Onuma Y, Ormiston J, Abizaid A, Waksman R, Serruys P. Bioresorbable scaffolds: rationale, current status, challenges, and future. Eur Heart J 2014;35:765-776.

13. Moussa ID, Klein LW, Shah B, et al. Consideration of a new definition of clinically relevant myocardial infarction after coronary revascularization: an expert consensus document from the Society for Cardiovascular Angiography and Interventions (SCAI). J Am Coll Cardiol 2013;62:1563-1570.

14. Cutlip DE, Windecker S, Mehran R, et al. Clinical end points in coronary stent trials: a case for standardized definitions. Circulation 2007;115:2344-2351.

15. Kereiakes DJ, Ellis SG, Popma JJ, et al. Evaluation of a fully bioresorbable vascular scaffold in patients with coronary artery disease: design of and rationale for the ABSORB III randomized trial. Am Heart J 2015;170:641-651.

16. Lane JP, Perkins LE, Sheehy AJ, et al. Lumen gain and restoration of pulsatility after implantation of a bioresorbable vascular scaffold in porcine coronary arteries. JACC Cardiovasc Interv 2014;7:688-695.

17. Serruys PW, Ormiston JA, Onuma Y, et al. A bioabsorbable everolimus-eluting coronary stent system (ABSORB): 2-year outcomes and results from multiple imaging methods. Lancet 2009;373:897-910.

18. Kraak RP, Hassell ME, Grundeken MJ, et al. Initial experience and clinical evaluation of the Absorb bioresorbable vascular scaffold (BVS) in real-world practice: the AMC Single Centre Real World PCI Registry. EuroIntervention 2015;10:1160-1168.

19. Capodanno D, Gori T, Nef H, et al. Percutaneous coronary intervention with everolimus-eluting bioresorbable vascular scaffolds in routine clinical practice: early and midterm outcomes from the European multicenter GHOSTEU registry. EuroIntervention 2015;10:1144-1153.

20. Kawamoto H, Panoulas VF, Sato K, et al. Impact of strut width in periprocedural myocardial infarction: a propensity-matched comparison between bioresorbable scaffolds and the first-generation sirolimus-eluting stent. JACC Cardiovasc Interv 2015;8:900-909.

21. Ishibashi Y, Muramatsu T, Nakatani S, et al. Incidence and potential mechanism(s) of post-procedural rise of cardiac biomarker in patients with coronary artery narrowing after implantation of an everolimus-eluting bioresorbable vascular scaffold or everolimus-eluting metallic stent. JACC Cardiovasc Interv 2015;8:1053-1063.

22. Teeuwen K, Hubbers S, Tijssen JG, Van Der Heyden JA, Rensing BJ, Suttorp MJ. Experiences with the Absorb everolimus-eluting bioresorbable vascular scaffold in all comers: The St. Antonius Hospital single centre registry. Clin Trials Regul Sci Cardiol 2015;10:1-6.

23. Holmes DR Jr, Kereiakes DJ, Garg S, et al. Stent thrombosis. J Am Coll Cardiol 2010;56:1357-1365.

24. Colombo A, Ruparelia N. Who is thrombogenic: the scaffold or the doctor? Back to the future! JACC Cardiovasc Interv 2016;9:25-27.

25. Allahwala UK, Cockburn JA, Shaw E, Figtree GA, Hansen PS, Bhindi R. Clinical utility of optical coherence tomography (OCT) in the optimisation of Absorb bioresorbable vascular scaffold deployment during percutaneous coronary intervention. EuroIntervention 2015;10:1154-1159.

26. Lee SW, Park SW, Hong MK, et al. Triple versus dual antiplatelet therapy after coronary stenting: impact on stent thrombosis. J Am Coll Cardiol 2005;46:1833-1837. 